\title{
HEAVY MESON MOLECULES IN EFFECTIVE FIELD THEORY
}

\author{
MOHAMMAD T. ALFIKY, FABRIZIO GABBIANI, ALEXEY A. PETROV* \\ Department of Physics and Astronomy, Wayne State University, \\ Detroit, Michigan, 48201, USA \\ *Presenter.E-mail: apetrov@wayne.edu \\ www.physics.wayne.edu/ apetrov/
}

\begin{abstract}
We consider the implications from the possibility that the recently observed state $X(3872)$ is a meson-antimeson molecule. We write an effective Lagrangian consistent with the heavy-quark and chiral symmetries needed to describe $X(3872)$. We explore the consequences of the assumption that $X(3872)$ is a molecular bound state of $D^{* 0}$ and $\bar{D}^{0}$ mesons for the existence of bound states in the $D^{0} \bar{D}^{0}$ and $D^{* 0} \bar{D}^{* 0}$
\end{abstract}

\section{Introduction}

The unusual properties of $X(3872)$ state, recently discovered in the decay $X(3872) \rightarrow J / \psi \pi^{+} \pi^{-}$, invited some speculations regarding its possible non$c \bar{c}$ nature ${ }^{1,2}$. Since its mass lies tantalizingly close to the $D^{* 0} \bar{D}^{0}$ threshold of $3871.3 \mathrm{MeV}$, it is tempting to interpret $X(3872)$ as a $D^{* 0} \bar{D}^{0}$ molecule with $J^{P C}=1^{++}$quantum numbers ${ }^{3,4}$. Such molecular states can be studied using techniques of effective field theories (EFT). This study is possible due to the multitude of scales present in QCD. The extreme smallness of the binding energy, $E_{b}=\left(m_{D^{0}}+m_{D^{0 *}}\right)-M_{X}=-0.6 \pm 1.1 \mathrm{MeV}$, suggests that this state can play the role of the "deuteron" 3 in mesonantimeson interactions. This fact allows us to use methods similar to those developed for the description of the deuteron ${ }^{5,6}$, with the added benefit of heavy-quark symmetry. A suitable effective Lagrangian describing such a system contains only heavy-meson degrees of freedom with interactions approximated by local four-boson terms constrained only by the symmetries of the theory. While the predictive power of this approach is somewhat limited, several model-independent statements can be made. For instance, possible existence of a molecular state in $D^{* 0} \bar{D}^{0}$ channel does not imply a molecular state in $D^{* 0}{\overline{D^{*}}}^{0}$ or $D^{0} \bar{D}^{0}$ channels. 
The tiny binding energy of this molecular state introduces an energy scale which is much smaller than the mass of the lightest particle, the pion, whose exchange can provide binding. This fact presents a problem with a straightforward EFT analysis of this bound state, which can be illustrated in the following example. Consider a low-energy s-wave scattering amplitude $A(k)$ of two heavy mesons with momentum $k$ (ignore spin for a moment),

$$
A(k)=\frac{4 \pi}{m_{D}} \frac{1}{k \cot \delta-i k}=\frac{4 \pi}{m_{D}} \frac{1}{(-1 / a)+\left(r_{0} / 2\right) k^{2}+\ldots-i k},
$$

where $a$ is a scattering length, which is related to the binding energy $E_{b}$ of a meson-antimeson bound state as $a \sim\left(E_{b}\right)^{-1 / 2}$. Naturally, $a \sim 1 / m$, where $m$ is the mass of exchanged particle that provides binding. Scattering amplitude of Eq. (1) can be obtained from an effective Lagrangian expressed in terms of only heavy-meson degrees of freedom as a power series in momentum $k$,

$$
A(k)=\frac{4 \pi}{m_{D}} \sum_{n} C_{n} k^{n}=-\frac{4 \pi a}{m_{D}}\left[1-i a k+\frac{a r_{0}-2 a^{2}}{2} k^{2}+\ldots\right],
$$

where $C_{n}$ are the coefficients of that effective Lagrangian. A problem with a simple application of EFT is apparent, as $a \rightarrow \infty$ for $E_{b} \rightarrow 0$, making the series convergence in Eq. (2) problematic. Indeed, $a \simeq 0.032 \mathrm{MeV}^{-1}$ for $\mathrm{X}(3872)$, which is much larger than the inverse masses of possible exchange particles, $1 / m_{\pi} \simeq 7.1 \times 10^{-3} \mathrm{MeV}^{-1}, 1 / m_{\rho} \simeq 1.3 \times 10^{-3} \mathrm{MeV}^{-1}$, etc. This implies that all-order resummation of $(a k)^{n}$ series is required. In EFT language this would imply resummation of a class of "bubble" graphs, whose vertices are defined by $C_{n}$.

\section{The effective Lagrangian}

The general effective Lagrangian required for description of $D^{* 0} \bar{D}^{0}$ molecular state and consistent with heavy-quark spin and chiral symmetries can be written as ${ }^{7}$

$$
\mathcal{L}=\mathcal{L}_{1}+\mathcal{L}_{2},
$$

where the two-body piece $\mathcal{L}_{2}$ describes the interactions between heavy meson degrees of freedom. The one-body piece $\mathcal{L}_{1}$ describes strong interactions of the heavy mesons $P$ and $P^{*}(P=B, D)$ containing one heavy quark $Q$ and is well known ${ }^{8}$ :

$$
\mathcal{L}_{1}=-\operatorname{Tr}\left[\bar{H}^{(Q)}\left(i v \cdot D+\frac{D^{2}}{2 m_{P}}\right) H^{(Q)}\right]+\frac{\lambda_{2}}{m_{P}} \operatorname{Tr}\left[\bar{H}^{(Q)} \sigma^{\mu \nu} H^{(Q)} \sigma_{\mu \nu}\right]+.(4)
$$


where the ellipsis denotes higher-order terms in chiral expansion, or describing pion- $H$ interactions and antimeson degrees of freedom $H_{a}^{(\bar{Q})}$ and $H_{a}^{(\bar{Q}) \dagger}$. A superfield describing the doublet of pseudoscalar heavy-meson fields $P_{a}=\left(P^{0}, P^{+}\right)$and their vector counterparts with $v \cdot P_{a}^{*(Q)}=0$, is defined as $H_{a}^{(Q)}=(1 / 2)(1+/ v)\left[P_{a \mu}^{*(Q)} \gamma^{\mu}-P_{a}^{(Q)} \gamma_{5}\right]$. The second term in Eq. (4) accounts for the $P-P^{*}$ mass difference $\Delta \equiv m_{P^{*}}-m_{P}=-2 \lambda_{2} / m_{P}$. The two-body piece is ${ }^{7}$

$$
\begin{aligned}
\mathcal{L}_{2}= & -\frac{C_{1}}{4} \operatorname{Tr}\left[\bar{H}^{(Q)} H^{(Q)} \gamma_{\mu}\right] \operatorname{Tr}\left[H^{(\bar{Q})} \bar{H}^{(\bar{Q})} \gamma^{\mu}\right] \\
& -\frac{C_{2}}{4} \operatorname{Tr}\left[\bar{H}^{(Q)} H^{(Q)} \gamma_{\mu} \gamma_{5}\right] \operatorname{Tr}\left[H^{(\bar{Q})} \bar{H}^{(\bar{Q})} \gamma^{\mu} \gamma_{5}\right] .
\end{aligned}
$$

Heavy-quark spin symmetry implies that the same Lagrangian governs the four-boson interactions of all $P_{a}^{(*)}=D^{(*)}$ states. Indeed, not all of these states are bound. Here we shall concentrate on $X(3872)$, which we assume to be a bound state of two neutral bosons, $P_{a} \equiv P^{0} \equiv D^{3}$. Evaluating the traces yields for the $D \overline{D^{*}}$ sector

$$
\begin{aligned}
\mathcal{L}_{2, D D^{*}}= & -C_{1} D^{(c) \dagger} D^{(c)} D_{\mu}^{*(\bar{c}) \dagger} D^{*(\bar{c}) \mu}-C_{1} D_{\mu}^{*(c) \dagger} D^{*(c) \mu} D^{(\bar{c}) \dagger} D^{(\bar{c})} \\
& +C_{2} D^{(c) \dagger} D_{\mu}^{*(c)} D^{*(\bar{c}) \dagger \mu} D^{(\bar{c})}+C_{2} D_{\mu}^{*(c) \dagger} D^{(c)} D^{(\bar{c}) \dagger} D^{*(\bar{c}) \mu}+\ldots(6)
\end{aligned}
$$

As we show later, the resulting binding energy depends on a linear combination of $C_{1}$ and $C_{2}$. Similarly, one obtains the component Lagrangian governing the interactions of $D$ and $\bar{D}$,

$$
\mathcal{L}_{2, D D}=C_{1} D^{(c) \dagger} D^{(c)} D^{(\bar{c}) \dagger} D^{(\bar{c})} .
$$

Clearly, one cannot relate the existence of the bound state in the $D \overline{D^{*}}$ and $D \bar{D}$ channels, as the properties of the latter will depend only on $C_{1}$.

\section{Properties of bound states}

The lowest-energy bound state of $D$ and $\overline{D^{*}}$ is an eigenstate of charge conjugation,

$$
\left|X_{ \pm}\right\rangle=\frac{1}{\sqrt{2}}\left[\left|D^{*} \bar{D}\right\rangle \pm\left|D \bar{D}^{*}\right\rangle\right] .
$$

To find the bound-state energy of $X(3872)$ with $J^{P C}=1^{++}$, we shall look for a pole of the transition amplitude $T_{++}=\left\langle X_{+}|T| X_{+}\right\rangle$. Defining $D D^{*}$ $D D^{*}$ transition amplitudes,

$$
\begin{array}{ll}
T_{11}=\left\langle D^{*} \bar{D}|T| D^{*} \bar{D}\right\rangle, & T_{12}=\left\langle D^{*} \bar{D}|T| D^{*}\right\rangle, \\
T_{21}=\left\langle D \bar{D}^{*}|T| D^{*} \bar{D}\right\rangle, & T_{22}=\left\langle D \bar{D}^{*}|T| D \bar{D}^{*}\right\rangle,
\end{array}
$$


we also have to include a "bubble" resummation of loop contributions, as existence of a bound state is related to a breakdown of perturbative expansion ${ }^{5}$. These amplitudes satisfy a system of Lippmann-Schwinger equations ${ }^{7}$. In an algebraic matrix form,

$$
\left(\begin{array}{l}
T_{11} \\
T_{12} \\
T_{21} \\
T_{22}
\end{array}\right)=\left(\begin{array}{c}
-C_{1} \\
C_{2} \\
C_{2} \\
-C_{1}
\end{array}\right)+i \widetilde{A}\left(\begin{array}{cccc}
-C_{1} & C_{2} & 0 & 0 \\
C_{2} & -C_{1} & 0 & 0 \\
0 & 0 & -C_{1} & C_{2} \\
0 & 0 & C_{2} & -C_{1}
\end{array}\right)\left(\begin{array}{c}
T_{11} \\
T_{12} \\
T_{21} \\
T_{22}
\end{array}\right) .
$$

The solution of Eq. (10) produces the $T_{++}$amplitude,

$$
T_{++}=\frac{1}{2}\left(T_{11}+T_{12}+T_{21}+T_{22}\right)=\frac{\lambda}{1-i \lambda \widetilde{A}},
$$

where $\lambda=C_{2}-C_{1}$ and $\widetilde{A}$ is a (divergent) integral

$$
\begin{array}{r}
\widetilde{A}=\frac{i}{4} 2 \mu_{D D^{*}} \int \frac{d^{3} q}{(2 \pi)^{3}} \frac{1}{\vec{q}^{2}-2 \mu_{D D^{*}}(E-\Delta)-i \epsilon} \\
=-\frac{1}{8 \pi} \mu_{D D^{*}}|\vec{p}| \sqrt{1-\frac{2 \mu_{D D^{*}} \Delta}{\vec{p}^{2}}} .
\end{array}
$$

Here $E=\vec{p}^{2} / 2 \mu_{D D^{*}}$, with $\mu_{D D^{*}}$ being the reduced mass of the $D D^{*}$ system. The divergence of the integral of Eq. (12) is removed by renormalization. We chose to define a renormalized $\lambda_{R}$ within the $M S$ subtraction scheme in dimensional regularization, which does not introduce any new dimensionfull scales into the problem. In this scheme the integral $\widetilde{A}$ is finite, which corresponds to an implicit subtraction of power divergences in Eq. (12). This implies for the transition amplitude

$$
T_{++}=\frac{\lambda_{R}}{1+(i / 8 \pi) \lambda_{R} \mu_{D D^{*}}|\vec{p}| \sqrt{1-2 \mu_{D D^{*}} \Delta / \vec{p}^{2}}} .
$$

The position of the pole of the molecular state on the energy scale can be read off Eq. (13),

$$
E_{\text {Pole }}=\frac{32 \pi^{2}}{\lambda_{R}^{2} \mu_{D D^{*}}^{3}}-\Delta .
$$

Recalling the definition of binding energy $E_{b}$ and that $m_{D^{*}}=m_{D}+\Delta$, we infer

$$
E_{b}=\frac{32 \pi^{2}}{\lambda_{R}^{2} \mu_{D D^{*}}^{3}}
$$




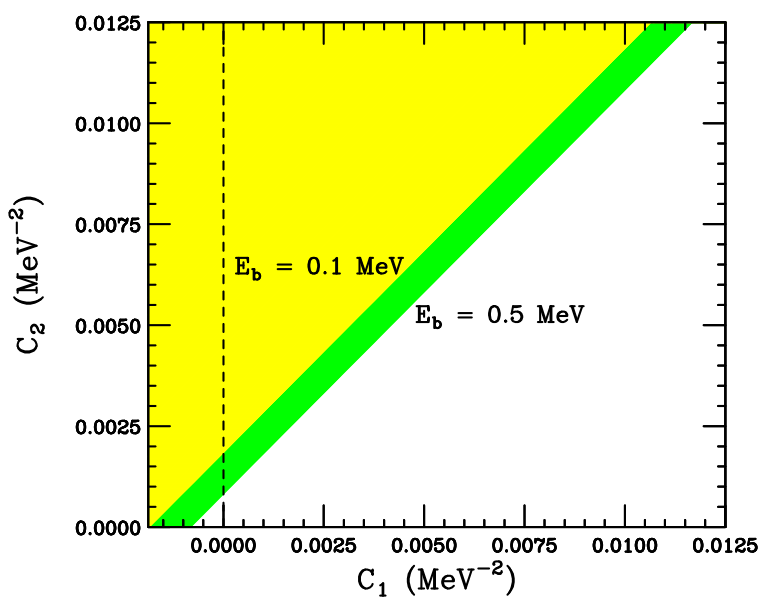

Fig. 1. The coupling constant $C_{2}$ is plotted vs. $C_{1}$. The lightly shaded area shows the region of parameter space allowed by postulating the existence of a $J^{P C}=1^{++}$bound state with $E_{b}=0.1 \mathrm{MeV}$, while excluding the orthogonal bound state with $\mathrm{C}=-1$. The darker area becomes allowed in addition if we assume $E_{b}=0.5 \mathrm{MeV}$.

Assuming $E_{b}=0.5 \mathrm{MeV}$, which is one sigma below the central value ${ }^{2}$, and the experimental values for the masses ${ }^{9}$, we obtain $\lambda_{R} \simeq 8.4 \times 10^{-4} \mathrm{MeV}^{-2}$.

Similar considerations apply to $D^{0} \bar{D}^{0}$ state, in which case the starting point is the Lagrangian term in Eq. (7). Since it involves only a single term, the calculations are actually easier and involve only one LippmannSchwinger equation. The resulting binding energy is then ${ }^{7}$

$$
E_{b}=\frac{256 \pi^{2}}{C_{1 R}^{2} m_{D}^{3}} .
$$

Examining Eq. (16) we immediately notice that the existence of a bound state in the $D^{*} \bar{D}$ channel does not dictate the properties of a possible bound state in the $D^{0} \bar{D}^{0}$ or $B^{0} \bar{B}^{0}$ channels, since $C_{1}$ and $C_{2}$ are generally not related to each other.

If we assume that the orthogonal state with $J^{P C}=1^{+-}$is not bound, which is consistent with all the existing experimental observations, we can place some separate constraints on the renormalized values of $C_{1}$ and $C_{2}$. The amplitude orthogonal to $T_{++}$,

$$
T_{--}=\left\langle X_{-}|T| X_{-}\right\rangle=\frac{1}{2}\left(T_{11}-T_{12}+T_{21}-T_{22}\right)=\frac{\lambda_{R}^{\prime}}{1-i \lambda_{R}^{\prime} \widetilde{A}_{R}},
$$

with $\lambda_{R}^{\prime}=-C_{1}-C_{2}$, does not have a pole that corresponds to a bound state if $C_{1}+C_{2}>0$. The exclusion of the $C=-1$ state together with 
the assumption of the existence of the $C=+1$ state limits the $\left(C_{1}, C_{2}\right)$ parameter space as shown in Fig. 1.

\section{Conclusions}

We introduced an effective field theory approach in the analysis of the likely molecular state $X(3872)$. We described its binding interaction with contact terms in a heavy-quark symmetric Lagrangian. The flexibility of this description allows us to ignore the details of the interaction and to concentrate on its effects, namely a shallow bound state and a large scattering length. We found that the existence of the bound state in the $D^{*} \bar{D}$ channel does not in general exclude a possibility of a bound state in the $D^{0} \bar{D}^{0}$ system, but does not require it. Future experimental studies of this state are interesting ${ }^{10}$ and should provide lots of new information about properties of QCD bound states.

This work was supported in part by the U.S. Department of Energy under Contract DE-FG02-96ER41005. A.P. was also supported by the U.S. National Science Foundation CAREER Award PHY-0547794.

\section{References}

1. For a review of properties of $\mathrm{X}(3872)$, see E. S. Swanson, Phys. Rept. 429, 243 (2006); C. Quigg, Nucl. Phys. Proc. Suppl. 142, 87 (2005); S. L. Olsen [Belle Collaboration], Int. J. Mod. Phys. A 20, 240 (2005). Possible interpretations of X(3872) also include a "hybrid charmonium" state: A. A. Petrov, proceeding of the 1st Meeting of the APS Topical Group on Hadronic Physics (GHP2004), Batavia, Illinois, 24-26 Oct 2004, J. Phys. Conf. Ser. 9, 83 (2005); see also G. Chiladze, A. F. Falk, and A. A. Petrov, Phys. Rev. D 58, 034013 (1998); F. E. Close and S. Godfrey, Phys. Lett. B 574, 210 (2003); B. A. Li, Phys. Lett. B 605, 306 (2005), a diquark-antidiquark state: L. Maiani, F. Piccinini, A. D. Polosa, and V. Riquer, Phys. Rev. D 71, 014028 (2005), a glueball: K. K. Seth, Phys. Lett. B 612, 1 (2005), and a cusp feature associated with the $D^{0} \bar{D}^{0}$ threshold: D. V. Bugg, Phys. Lett. B 598, 8 (2004). See also T. Barnes and S. Godfrey, Phys. Rev. D 69, 054008 (2004); E. J. Eichten, K. Lane, and C. Quigg, Phys. Rev. D 69, 094019 (2004); T. Barnes, S. Godfrey, and E. S. Swanson, arXiv:hep-ph/0505002. Some older studies include M. B. Voloshin and L. B. Okun, JETP Lett. 23, 333 (1976) [Pisma Zh. Eksp. Teor. Fiz. 23, 369 (1976)]; A. De Rujula, H. Georgi, and S. L. Glashow, Phys. Rev. Lett. 38, 317 (1977). S. Nussinov and D. P. Sidhu, Nuovo Cim. A 44, 230 (1978); N. A. Törnqvist, Z. Phys. C 61, 525 (1994); A. V. Manohar and M. B. Wise, Nucl. Phys. B 399, 17 (1993).

2. S. K. Choi et al. [Belle Collaboration], Phys. Rev. Lett. 91, 262001 (2003); B. Aubert et al. [BABAR Collaboration], Phys. Rev. D 71, 071103 (2005); 
D. Acosta et al. [CDF II Collaboration], Phys. Rev. Lett. 93, 072001 (2004); V. M. Abazov et al. [D0 Collaboration], Phys. Rev. Lett. 93, 162002 (2004).

3. N. A. Törnqvist, Phys. Lett. B 590, 209 (2004); C. Y. Wong, Phys. Rev. C 69, 055202 (2004). F. Close and P. Page, Phys. Lett. B 578, 119 (2004); E. S. Swanson, Phys. Lett. B 588, 189 (2004).

4. E. Braaten and M. Kusunoki, Phys. Rev. D 69, 074005 (2004).

5. S. Weinberg, Nucl. Phys. B 363, 3 (1991); Phys. Lett. B 251, 288 (1990).

6. D. B. Kaplan, M. J. Savage and M. B. Wise, Nucl. Phys. B 534, 329 (1998); ibid Phys. Lett. B 424, 390 (1998).

7. M. T. AlFiky, F. Gabbiani and A. A. Petrov, Phys. Lett. B 640, 238 (2006).

8. A. V. Manohar and M. B. Wise, "Heavy quark physics," Camb. Monogr. Part. Phys. Nucl. Phys. Cosmol. 10, 1 (2000).

9. S. Eidelman et al., Phys. Lett. B592, 1 (2004).

10. M. B. Voloshin, Phys. Lett. B 604, 69 (2004); E. Braaten and M. Kusunoki, Phys. Rev. D 71, 074005 (2005); arXiv:hep-ph/0506087; E. Braaten, M. Kusunoki, and S. Nussinov, Phys. Rev. Lett. 93, 162001 (2004); E. Braaten and M. Lu, arXiv:hep-ph/0606115; S. Pakvasa and M. Suzuki, Phys. Lett. B 579, 67 (2004); J. L. Rosner, Phys. Rev. D 70, 094023 (2004). 